\title{
Effect of Advanced Airway Management by Paramedics During Out-of-Hospital Cardiac Arrest on Chest Compression Fraction and Return of Spontaneous Circulation
}

\section{Koji Shimizu \\ Masahiro Wakasugi (D) \\ Toshiomi Kawagishi \\ Tomoya Hatano \\ Takamasa Fuchigami \\ Hiroshi Okudera}

Emergency and Disaster Medicine, University of Toyama, Toyama, Japan
Correspondence: Masahiro Wakasugi Emergency and Disaster Medicine,

University of Toyama, 930-0194 Sugitani, Toyama, Japan

Tel +8I 76-434-7786

$\mathrm{Fax}+81$ 76-434-5II0

Email mwaka@med.u-toyama.ac.jp
Purpose: To obtain effective systemic blood flow and coronary perfusion by chest compressions during cardiopulmonary resuscitation, it is recommended that the interruption time of chest compressions be kept to a minimum, and that the chest compression fraction (CCF) should be kept high. In this study, we examined the effects of advanced airway management by paramedics in out-of-hospital cardiac arrest (OHCA) cases on CCF and on return of spontaneous circulation (ROSC) before arrival at the hospital.

Participants and Methods: A total of 283 adult, non-traumatic OHCA cases who were in cardiopulmonary arrest at the time of EMS contact between April 2015 and March 2017 were registered for this study. We retrospectively investigated the presence or absence of advanced airway clearance, $\mathrm{CCF}$ and ROSC during CPR. CCF was calculated by measuring the chest compression interruption time from the ECG waveform recorded on a semiautomatic defibrillator (Philips HeartStart MRX). The data obtained were recorded on a computer, and comparisons between groups were examined using an untested $t$-test and $\chi^{2}$ - test.

Results: Of the 283 patients with OHCA, 159 were included in the analysis. The CCF of the AAM group was $89.4 \%$, which was significantly higher than that in the BMV group $(84.3 \%)$ $(\mathrm{P}<0.01)$. Forty-one patients had ROSC at the time of arrival at the hospital, and the CCF of ROSC patients was $89.7 \%$, which was significantly higher than that of non-ROSC patients $(87.2 \%)(\mathrm{P}<0.01)$. ROSC was also obtained in $31.8 \%$ of the patients in the AAM group, which was significantly higher than that in the BMV group (12.2\%).

Conclusion: In this study, we found that advanced airway management during prehospital emergency transport by paramedics showed high CCF and ROSC rates and contributed to improving the prognosis of OHCA patients through high-quality resuscitation.

Keywords: cardio pulmonary resuscitation, endotracheal intubation, supraglottic airways devices, outcome

\section{Introduction}

More than 120,000 out-of-hospital cardiac arrest (OHCA) victims were transported to the emergency room in Japan in 2015. Their one-month survival rates are low at $<10 \%,{ }^{1}$ and improving prognosis of these cases is an urgent issue. "High-quality CPR" is key to improving the chance of survival for OHCA patients. Maintaining a high chest compression fraction (CCF; percentage of time patients received compressions through constant chest compressions) is considered a key factor in achieving return of spontaneous circulation (ROSC) of OHCA resuscitation. ${ }^{2,3}$ 
American Heart Association guidelines for cardiopulmonary resuscitation recommend maintaining the $\mathrm{CCF}$ above $80 \%$ during CPR. ${ }^{4}$

During prehospital resuscitation, rescuers may stop chest compressions for many reasons that are integral to patient care, such as ventilation, assessing heart rhythm and pulse, defibrillation, or tracheal intubation. Among them, the interruption time of chest compressions for ventilation may have a significant impact on CCF. ${ }^{5}$ Two methods are used for chest compressions and ventilation during cardiopulmonary resuscitation: synchronous CPR, a cycle of 30 uninterrupted chest compressions with a cycle of two ventilations or asynchronous CPR, and uninterrupted ventilation with advanced airway management. Interruption for ventilation as synchronous CPR may have a significant impact on CCF. Advanced airway management with endotracheal intubation (ETI) or supraglottic airway devices (SGA) allows ventilation without interruption of chest compressions and is expected to increase CCF. However, the relationship between the effect of airway management in OHCA on actual CCF and patient outcomes is unknown. It has also been pointed out that ETI by paramedics in hospitals may worsen the prognosis of cardiac arrest patients ${ }^{6,7}$ The purpose of this study was to determine the optimal airway management strategy for prehospital care by paramedics by investigating the impact of prehospital advanced airway management on CCF and ROSC in OHCA patients.

\section{Materials and Methods Study Design}

This study included data from patients who experienced out-of-hospital cardiac arrest in Hakusan-Nonoich region, Ishikawa, Japan between April 2015 and March 2017. Patients who were already experiencing cardiac arrest at the time of EMS arrival and intervention were included in the study. All data were retrospectively collected from the Utstein style database of the Emergency Medical Services (EMS) of the Hakusan-Nonoichi fire department. Patient data was kept confidential.

Three to four emergency medical technicians (EMTs) in one ambulance were dispatched to the cardiac arrest event. At least one of the EMTs was a paramedic who was able to provide defibrillation, intravenous adrenaline, and advanced airway management by tracheal intubation or, alternatively, by supraglottic devices. Patients were transported to tertiary care hospitals, where standardized post- resuscitation care was provided according to predefined protocols. EMTs provided CPR according to the standard guidelines with emphasis on high-quality chest compressions (rate 100-120 min-1, depth 5-6 cm, allowing full recoil of chest after each compression, and minimizing interruptions). The defibrillator pads were promptly connected to check the rhythm and defibrillated if necessary. Before applying ETI or SGA, a synchronized 30-to-2 compressions/ventilation ratio was attained, with ventilation provided by a bag-valve-mask (BVM) with minimalized interruptions (within 10 seconds). The choice of airway management strategy was left to the judgment of paramedics. In cases where standard BMV was impossible, or in case of massive regurgitation of gastric contents during ventilation, ETI or SGA were recommended. After intubation, ventilation was delivered independently and asynchronously during uninterrupted chest compressions. Regardless of whether ETI or SGA was used, the time to secure the airway was always within 10 seconds of the cessation of chest compressions. No patients received mechanical CPR.

In this study, we compared the CCF during the entire active period and the ROSC rate before arrival at the hospital in two groups: the group ventilated only with BVM before the hospital (group BMV) and the group ventilated by the advanced airway (group AAM). The study was conducted in accordance with the guidelines outlined in the Declaration of Helsinki and study design was approved by an ethics review board of University of Toyama. Our ethical committee waived the requirement for obtaining informed consent from patients because of the retrospective and observational setting of the research.

\section{Data Analysis}

For the calculation of $\mathrm{CCF}$, the chest compression interruption time was measured from the ECG waveform recorded using a semiautomatic defibrillator (Philips HeartStart MRX) during emergency service activities. A regular waveform with a constant amplitude (roughly 100-120 times/min) was judged to be a chest compression waveform, and a time when only the ventricular fibrillation (Vf) waveform or other irregular baseline oscillations were seen was judged to be a chest compression interruption time. Measurements were taken twice by two researchers, and the average of the measurements was considered for analysis. Cases were excluded from the study when both researchers judged that chest compression time could not be measured from ECG recordings. 
Chest compression time was calculated by subtracting the chest compression interruption time from the emergency operation time from the time of arrival at the patient's side to the time of arrival at the hospital, dividing it by the emergency operation time. Categorical data were reported as percentages and compared using the chi-square test. Quantitative variables were reported as medians with interquartile ranges (IQR) and were analyzed using the MannWhitney test. We used multivariable logistic regression to correct the results for the difference in baseline characteristics of the patients. Statistical significance was set at $\mathrm{P}<0.05$. Statistical analyses were performed using the $\mathrm{JMP}^{\circledR}$ Pro 14.2.0.

\section{Results}

A total of 283 cardiac arrest victims were transported to the emergency room during the study period. After excluding patients who were considered ineligible for resuscitation, those that received first response by EMTs who could not use advanced airway devices, and those whose chest compression times could not be determined from the ECG records, 159 patients were included in the study. Advanced airway management was used in 110 of 159 patients (AAM group). The airway devices used were tracheal intubations in 50 cases, LTS in 58 cases, and i-gel in 2 cases. The remaining 49 cases were resuscitated using normal bag-mask ventilation (BMV). (Figure 1)

The AAM group was significantly older and had a higher rate of adrenalin use than the BVM group. There were no differences between the two groups in terms of sex or witnessed cardiac arrest, presence of bystander CPR, shockable rhythm, probable cause of cardiac arrest, training level of the responding ambulance crew, and duration of the resuscitation attempt. The CCF of the AAM group was $89.4 \%$, which was significantly higher than the $84.3 \%$ in the BMV group. ROSC was obtained in 41 patients $(25.8 \%)$ by the time they arrived at the hospital. In the AAM group, 31.8\% achieved ROSC, which was significantly higher than the $12.2 \%$ in the BMV group. (Table 1)

The CCF of ROSC patients was $89.7 \%$, which was significantly higher than that of non-ROSC patients $(87.2 \%)(\mathrm{P}<0.01)$. (Figure 2$)$

The logistic regression model was used to calculate the adjusted odds ratio (OR) with 95\% confidence interval (CI) for ROSC with adjustments for age, adrenaline administration, and CCF.
Both CCF and adrenaline administration were associated with achieved ROSC (OR 58.5, CI 4.43-1184, $\mathrm{p}=0.004$ and OR 4.4, CI 1.79-12.1, p=0.002, respectively), but age was not associated with achieved ROSC (OR 0.15, CI 0.02-1.32, $\mathrm{p}=0.09$ )

\section{Discussion}

This study was designed based on the hypothesis that advanced airway management during prehospital CPR by paramedics contributes to efficient ventilation, continuous chest compressions, and increased $\mathrm{CCF}$, which in turn contributes to improved outcomes for out-of-hospital cardiac arrest patients. Several previous studies have reported changes in CCF with the use of advanced airway devices; however, the results have not been consistent. Malinverni et al reported that patients who were intubated did not have an associated increase in CCF over the entire duration of resuscitation when compared to patients managed with bag mask ventilation. ${ }^{8}$ Other studies have shown that the use of the supraglottic airway during OHCA increases CCF when compared to standard bag mask ventilation ${ }^{9,10}$ Advanced airway management is advantageous in that it allows asynchronous ventilation during resuscitation and reduces interruption of chest compressions, ${ }^{11}$ the disadvantage is that, depending on the skill of the paramedic, securing the airway maneuver itself may take more time, which may in turn prolong the interruption of chest compressions. Differences in the style of pre-hospital rescue service and hospital transport times in countries and regions may have contributed to the variability in results. $^{12}$ In the present study, the effect of interruption of chest compressions by tracheal intubation or supraglottic airway devices was not significant, and it was proved that interruption of chest compressions by asynchronous ventilation using advanced airway devices had a more favorable effect on $\mathrm{CCF}$ in prehospital rescue activities in Japan.

In the present study, CCF was above the guideline recommended value ${ }^{4}$ in more than $80 \%$ of both $\mathrm{BMV}$ and AAM groups. Although the difference in $\mathrm{CCF}$ between the two groups was not large in absolute terms, it was statistically significant. The results of the regression analysis suggested that a high CCF may be associated with the achievement of ROSC, even after excluding confounding factors, suggesting that it is important to maintain a high CCF during CPR.

The relatively small difference in CCF between the two groups may be attributed to the short CPR time of about 


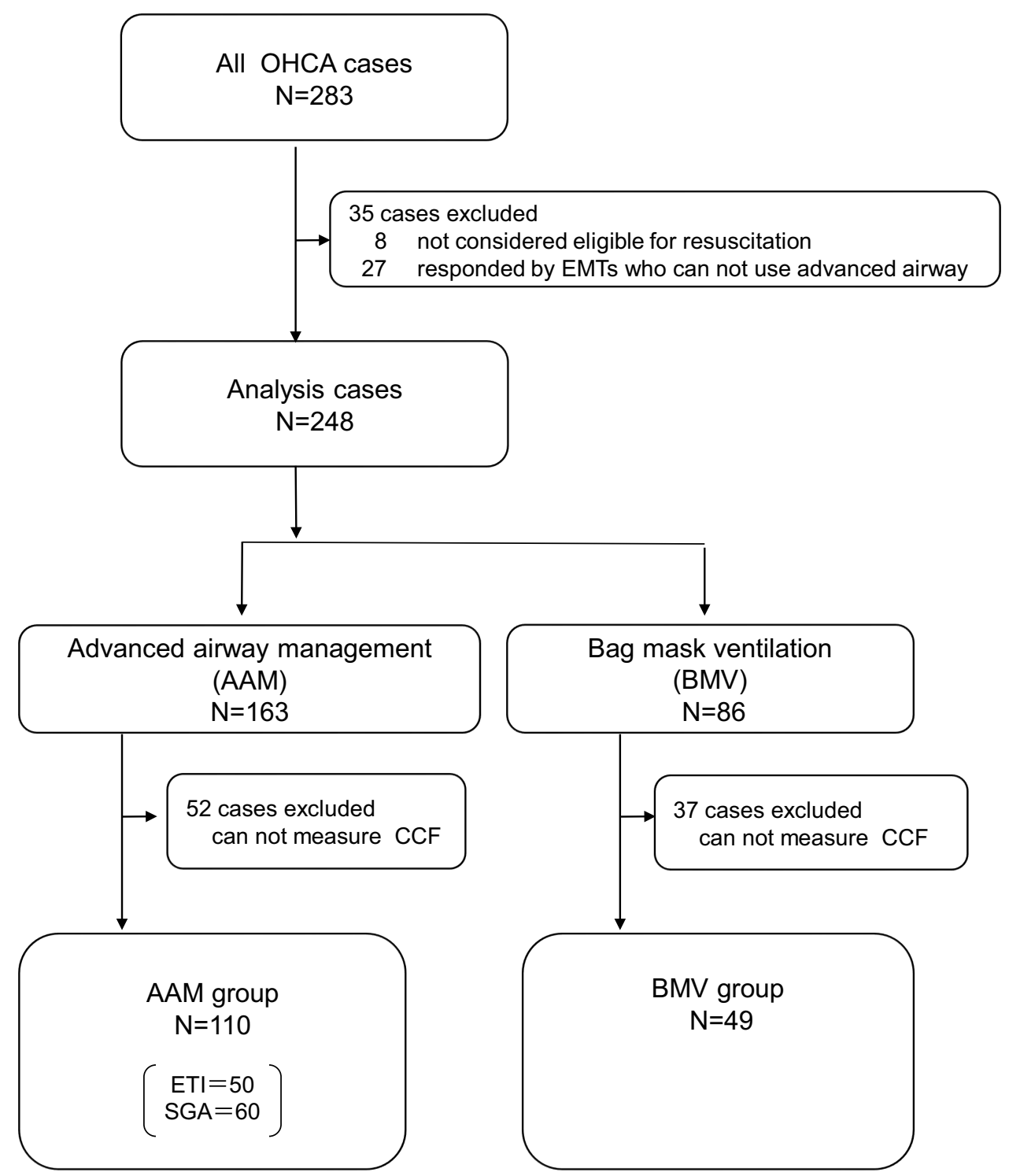

Figure I Flow diagram of study enrollment.

Abbreviations: OHCA, Out-of-Hospital Cardiac Arrest; EMT, emergency medical technician; CCF, chest compression fraction; ETI, end tracheal intubation; SGA, supraglottic airways

20 minutes. The effect of chest compression interruption time due to asynchronous ventilation may be more pronounced when performing longer CPR. Conversely, it has been reported that the high values of CCF are associated with low quality of CPR (especially the depth of chest compressions) and low survival rate, ${ }^{13}$ so the pros and cons of increasing CCF during prolonged CPR must be further investigated.

In recent years, randomized clinical studies have been conducted on the efficacy of various advanced airway securing devices for patients with OHCA. A randomized clinical trial on the effect of advanced airway clearance by paramedics on OHCA patients did not show the superiority of tracheal intubation compared with BVM ventilation. ${ }^{14}$ Low success rates for tracheal intubation by emergency services have been noted, which may have influenced these results. ${ }^{15,16}$ In the comparison between supraglottic devices and tracheal intubation for OHCA patient outcome, the evaluation is not consistent, with some reports showing a better prognosis with supraglottic devices ${ }^{17}$ and others showing no difference. ${ }^{18}$ There are no studies with a high level of evidence for a direct comparison between supraglottic devices and bag-mask ventilation. In our study, a higher ROSC rate was obtained in the group with AAM, including tracheal intubation and supraglottic devices, than in the group with BMV, indicating that the use of advanced airway devices by 
Table I Comparison of Characteristics of Patients, Chest Compression Fraction (CCF) and Return of Spontaneous Circulation (ROSC) Between Advanced Airway Management Group and Bag Mask Ventilation Group

\begin{tabular}{|c|c|c|c|}
\hline & $\begin{array}{l}\text { Advanced Airway Management AAM } \\
\qquad(n=110)\end{array}$ & $\begin{array}{l}\text { Bag Mask Ventilation BMV } \\
\qquad(n=49)\end{array}$ & p value \\
\hline Age, median (IQR) & $78(62-84)$ & $67(58-79)$ & 0.003 \\
\hline Sex (Men), No. (\%) & $60(54.5 \%)$ & $50(46.8 \%)$ & 0.261 \\
\hline Witnessed arrest, No (\%) & $36(32.7 \%)$ & $15(30.6 \%)$ & 0.792 \\
\hline Bystander resuscitation, No. (\%) & $66(60 \%)$ & $27(55.1 \%)$ & 0.563 \\
\hline $\begin{array}{l}\text { Presenting rhythm, No. (\%) } \\
\text { Asystole } \\
\text { Ventricular fibrillation/Pulseless VT } \\
\text { Pulseless electrical activity }\end{array}$ & $\begin{array}{c}83 \text { (75.5\%) } \\
3(2.7 \%) \\
24(21.8 \%)\end{array}$ & $\begin{array}{c}37(75.5 \%) \\
2(4.1 \%) \\
10(20.4 \%)\end{array}$ & 0.884 \\
\hline Etiology (cardiogenic), No. (\%) & $43(39.1 \%)$ & $23(47 \%)$ & 0.354 \\
\hline Time from call to EMS arrival, median (IQR) & $7 \min (6-8)$ & $7 \min (6-9)$ & 0.194 \\
\hline $\begin{array}{l}\text { Number of EMTs available for intubation, median } \\
\text { (IQR) }\end{array}$ & $2(I-2)$ & $2(I-2)$ & 0.076 \\
\hline Transfer Time to hospital, median (IQR) & $21 \min (15-25)$ & $18 \min (14-24)$ & 0.245 \\
\hline Adrenaline administration, No. (\%) & $74(67.3 \%)$ & $14(28.6 \%)$ & $<0.001$ \\
\hline Chest compression fraction, median (IQR) & $89.9 \%(88-91)$ & $84.5 \%(83-86)$ & $<0.001$ \\
\hline Return of spontaneous circulation, No (\%) & $35(31.8 \%)$ & $6(12.2 \%)$ & 0.006 \\
\hline
\end{tabular}

Abbreviations: IQR, interquartile range; VT, ventricular tachycardia; EMS, emergency medical service; EMT, emergency medical technician.

paramedics may improve prognosis. One reason for this could be the increase in the CCF caused by AAM.

However, a limitation of this study is its retrospective observational design, owing to which the possibility of selection bias in the choice of airway management method cannot
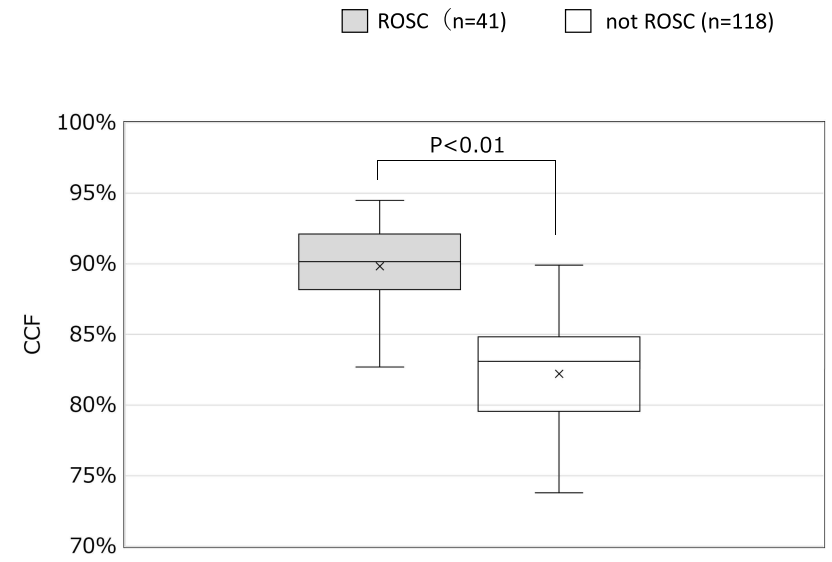

Figure 2 Comparison of chest compression fraction (CCF) between patients who achieved return of spontaneous circulation (ROSC) and those who did not (not ROSC). be ruled out. In this study, airway management technique was left to the discretion of each paramedic, so we cannot rule out the possibility that advanced airway management was preferentially selected in patients with a high probability of successful resuscitation. There may also be a confounding relationship between advanced airway management and adrenaline administration. Since it is difficult for a single person to manage the airway with bag-mask ventilation and simultaneously secure the intravenous route and administer drugs, adrenaline may have been administered only in cases where it was determined that there was a good chance of saving lives, and only after using an advanced airway. Another limitation is that we were not able to track longterm outcomes after hospitalization, therefore, we could not address the impact on final patient outcomes after hospital transport by EMS. It is necessary to accumulate more cases and investigate these issues in RCTs and other studies.

\section{Conclusion}

The use of advanced airway securing devices by paramedics for OHCA patients improves the quality of 
cardiopulmonary resuscitation by enabling high $\mathrm{CCF}$. Additionally, high ROSC rates can be obtained, which may contribute to improving the prognosis of patients with OHCS. The results of this study are important for future research on optimal airway management strategies in prehospital care.

\section{Disclosure}

The authors report no conflicts of interest in this work.

\section{References}

1. Kiguchi T, Okubo M, Nishiyama C, et al. Out-of-hospital cardiac arrest across the World: first report from the International Liaison Committee on Resuscitation (ILCOR). Resuscitation. 2020;152:39-49. doi:10.1016/j.resuscitation.2020.02.044

2. Vaillancourt C, Everson-Stewart S, Christenson J, et al. The impact of increased chest compression fraction on return of spontaneous circulation for out-of-hospital cardiac arrest patients not in ventricular fibrillation. Resuscitation. 2011;82(12):1501-1507. doi:10.1016/j. resuscitation.2011.07.011

3. Uppiretla AK, Gangalal GM, Rao S, Bosco DD, Shareef SM, Sampath V. Effects of chest compression fraction on return of spontaneous circulation in patients with cardiac arrest; a brief report. $A d v$ J Emerg Med. 2020;4(1):e8.

4. Meaney PA, Bobrow BJ, Mancini ME, et al. Cardiopulmonary resuscitation quality: [corrected] improving cardiac resuscitation outcomes both inside and outside the hospital: a consensus statement from the American Heart Association. Circulation. 2013;128(4):417-435. doi:10.1161/CIR.0b013e31829d8654

5. Jarman AF, Hopkins CL, Hansen JN, Brown JR, Burk C, Youngquist ST. Advanced Airway type and its association with chest compression interruptions during out-of-hospital cardiac arrest resuscitation attempts. Prehosp Emerg Care. 2017;21(5):628-635. doi:10.1080/10903127.2017.1308611

6. Shin SD, Ahn KO, Song KJ, Park CB, Lee EJ. Out-of-hospital airway management and cardiac arrest outcomes: a propensity score matched analysis. Resuscitation. 2012;83(3):313-319. doi:10.1016/j. resuscitation.2011.10.028

7. Hasegawa K, Hiraide A, Chang Y, Brown DF. Association of prehospital advanced airway management with neurologic outcome and survival in patients with out-of-hospital cardiac arrest. JAMA. 2013;309(3):257-266. doi:10.1001/jama.2012.187612
8. Malinverni S, Bartiaux M, Cavallotto F, et al. Does endotracheal intubation increases chest compression fraction in out of hospital cardiac arrest: a substudy of the CAAM trial. Resuscitation. 2019;137:35-40. doi:10.1016/j.resuscitation.2019.01.032

9. Maignan M, Koch FX, Kraemer M, et al. Impact of laryngeal tube use on chest compression fraction during out-of-hospital cardiac arrest. A prospective alternate month study. Resuscitation. 2015;93:113-117. doi:10.1016/j.resuscitation.2015.06.002

10. Kurz MC, Prince DK, Christenson J, et al. Association of advanced airway device with chest compression fraction during out-of-hospital cardiopulmonary arrest. Resuscitation. 2016;98:35-40. doi:10.1016/j. resuscitation.2015.10.011

11. Sanson G, Ristagno G, Caggegi GD, et al. Impact of 'synchronous' and 'asynchronous' CPR modality on quality bundles and outcome in out-of-hospital cardiac arrest patients. Intern Emerg Med. 2019;14 (7):1129-1137. doi:10.1007/s11739-019-02138-9

12. Oh YS, Ahn KO, Shin SD, et al. Variability in the effects of prehospital advanced airway management on outcomes of patients with out-of-hospital cardiac arrest. Clin Exp Emerg Med. 2020;7 (2):95-106. doi:10.15441/ceem.19.022

13. Baldi E, Contri E, Burkart R, et al. A Multicenter International Randomized Controlled Manikin Study on different protocols of cardiopulmonary resuscitation for laypeople: the MANI-CPR Trial. Simul Healthc. 2020. doi:10.1097/SIH.0000000000000505

14. Jabre P, Penaloza A, Pinero D, et al. Effect of bag-mask ventilation vs endotracheal intubation during cardiopulmonary resuscitation on neurological outcome after out-of-hospital cardiorespiratory arrest: a Randomized Clinical Trial. JAMA. 2018;319(8):779-787. doi:10.1001/jama.2018.0156

15. Katz SH, Falk JL. Misplaced endotracheal tubes by paramedics in an urban emergency medical services system. Ann Emerg Med. 2001;37 (1):32-37. doi:10.1067/mem.2001.112098

16. Wang HE, Yealy DM. How many attempts are required to accomplish out-of-hospital endotracheal intubation? Acad Emerg Med. 2006;13 (4):372-377. doi:10.1197/j.aem.2005.11.001

17. Wang HE, Schmicker RH, Daya MR, et al. Effect of a strategy of initial laryngeal tube insertion vs endotracheal intubation on 72-hour survival in adults with out-of-hospital cardiac arrest: a Randomized Clinical Trial. JAMA. 2018;320(8):769-778. doi:10.1001/ jama.2018.7044

18. Benger JR, Kirby K, Black S, et al. Effect of a strategy of a supraglottic airway device vs tracheal intubation during out-ofhospital cardiac arrest on functional outcome: the AIRWAYS-2 Randomized Clinical Trial. JAMA. 2018;320(8):779-791. doi:10.1001/jama.2018.11597
Open Access Emergency Medicine

\section{Publish your work in this journal}

The Open Access Emergency Medicine is an international, peerreviewed, open access journal publishing original research, reports, editorials, reviews and commentaries on all aspects of emergency medicine. The manuscript management system is completely online and includes a very quick and fair peer-review system, which is all easy to use. Visit http://www.dovepress.com/testimonials.php to read real quotes from published authors. 